\title{
Changes in Structural and Functional Responses of Bacterial Communities under Different Levels of Long-Term Compost Application in Paddy Soils ${ }^{\mathbb{S}}$
}

\author{
Sandipan Samaddar ${ }^{1 \dagger}$, Gwang Hyun Han $^{1 \dagger}$, Puneet Singh Chauhan ${ }^{2}$, Poulami Chatterjee ${ }^{1}$, Sunyoung Jeon ${ }^{1}$, \\ and Tongmin $\mathrm{Sa}^{1 *}$
}

${ }^{1}$ Department of Environmental and Biological Chemistry, Chungbuk National University, Cheongju 28644, Republic of Korea

${ }^{2}$ Division of Plant Microbe Interaction, CSIR-National Botanical Research Institute, Rana Pratap Marg, Lucknow, India

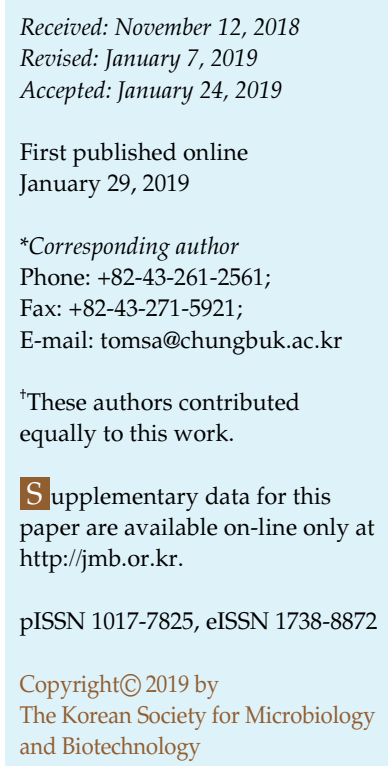

Soils amended for long-term with high levels of compost demonstrated greater abundance of bacterial members of the phylum Bacteroidetes whereas a decreasing trend in the relative abundance of phylum Acidobacteria was noted with increasing levels of compost. Metabolic profiles predicted by PICRUSt demonstrated differences in functional responses of the bacterial community according to the treatments. Soils amended with lower compost levels were characterized by abundance of genes encoding enzymes contributing to membrane transport and cell growth whereas genes encoding enzymes related to protein folding and transcription were enriched in soils amended with high levels of compost. Thus, the results of the current study provide extensive evidence of the influence of different compost levels on bacterial diversity and community structure in paddy soils.

Keywords: Bacterial diversity, long-term fertilization, compost levels, paddy soils
Experiments that monitor changes in microbial diversity and community structure over a span of decades are critical for predicting future soil productivity and understanding interactions in the soil environment. Soil microbial communities can be influenced by several biotic and abiotic factors [1-3], in addition to agricultural management practices, which include the use of organic/ inorganic fertilizers and crop rotation [4]. Several reports have indicated that long-term organic farming practices have positive effects on soil microbial diversity and soil processes $[5,6]$, but there is not much detailed evidence of how different levels or dosages of compost impact bacterial communities. In this study, we used pyro-sequencing tags between V1-V3 regions of the 16S rRNA gene to analyze and compare the effect of long-term application of different compost levels on the composition of paddy soil bacterial communities. Soil samples were collected from Gangseo series paddy soils at the National Institute of Agricultural Science and Technology experimental area located in Suwon city $\left(37^{\circ} 16^{\prime} 0^{\prime \prime} \mathrm{N}, 127^{\circ} 1^{\prime} 0^{\prime \prime} \mathrm{E}\right)$, Gyeonggi Province, Republic of Korea, where rice has been cultivated as a single crop since 1954 [7]. Five different treatments comprising inorganic chemical fertilizers and different levels of compost application were considered for the present study. The treatments included: (i) inorganic nitrogen $(\mathrm{N})$, phosphorus $(\mathrm{P})$ and potassium $(\mathrm{K})$ fertilizers (NPK), (ii) NPK and organic compost (C) (NPKC750), (iii) NPKC1500, (iv) NPKC2250 and (v) NPKC3000. Chemical fertilizers (NPK) were applied at different rates as N-P $\mathrm{O}_{5}$ $\mathrm{K}_{2} \mathrm{O}$ kilograms-per-hectare at the rate of 75-75-75 from 1954 to $1970,100-75-75$ from 1971 to $1978,150-86-86$ from 1979 to 1985 and 110-70-80 from 1986 until the sampling date. 
Table 1. Summary data for $16 \mathrm{~S}$ rRNA sequencing results from the samples used in this study.

\begin{tabular}{lcccccc}
\hline Treatments & $\begin{array}{c}\text { Number of sequences } \\
\text { after normalization }\end{array}$ & Sobs & $\begin{array}{c}\text { Goods coverage } \\
(\%)\end{array}$ & Shannon Index & Inv-simpson Index & Chao \\
\hline NPK & 787 & 168 & 96.05 & 4.81 & 109.32 & 185.42 \\
NPKC750 & 787 & 165 & 95.8 & 4.76 & 98.19 & 183.05 \\
NPKC1500 & 787 & 166 & 96.3 & 4.82 & 110.34 & 182.91 \\
NPKC2250 & 787 & 168 & 95.7 & 4.78 & 103.63 & 184.75 \\
NPKC3000 & 787 & 160 & 97.1 & 4.75 & 92.8 & 168.45 \\
\hline
\end{tabular}

The species observed (Sobs), coverage percentage (Good), richness estimators (Chao), and diversity indices (Shannon and inv-Simpson) were calculated using Mothur.

Organic compost was prepared by fermenting rice straw and then added at the rate of 7.5, 15.0, 22.5, and $30.0 \mathrm{Mg} \mathrm{ha}^{-1}$ in NPKC750, NPKC1500, NPKC2250, and NPKC3000 treatments, respectively. Three subsamples were collected from individual treatments at 0 to $20 \mathrm{~cm}$ depth in October 2009, after the rice crop harvest. Subsamples were pooled to make a composite sample, thus a total of 5 individual samples were collected. The physicochemical properties of the soils studied were as reported earlier [8]. DNA was extracted from approximately $0.5 \mathrm{~g}$ of soil using an UltraClean Soil DNA Isolation Kit (Mo Bio, Solana Beach, CA, USA), following the manufacturer's instructions. The extracted DNA was amplified using primers targeting the bacterial 16S rRNA gene (9F: 5'-X-ACG AGT TTG ATC MTG GCT CAG-3' and V3-541R: 5'-X-ACW TTA CCG CGG CTG CTG G-3', where $X$ denotes an 8-nucleotide-long barcode uniquely designed for each sample followed by a common linker AC), and were pyro-sequenced in a 454 GS FLX Titanium Sequencing System (Roche), according to the manufacturer's instructions at Chunlab, Seoul, South Korea. Raw data are available in the Mendeley data repository: http://dx.doi.org/10.17632/pgr2hc2d9k.1. Raw sequences obtained were analyzed as described [9]. After processing the raw sequences, they were clustered at $3 \%$ cut off level and a total of 188 OTUs were observed from all the studied samples. The rarefaction curve (Fig. S1) indicated that the sampling effort was sufficient to get a full extent of taxonomic diversity. This derives high importance as number of species found in a sample at any given phylogenetic level is strongly affected by the number of sequences analyzed [10]. After analyzing the sequence data and normalizing it to the minimum number of reads, a total of 787 sequences were obtained (Table 1). The number of OTUs observed ranged from 160-168 per sample and Goods coverage estimator demonstrated coverage ranging from 95.7-97.1\% which implies that the majority of phylotypes were considered for this study. The diversity estimators Shannon and Inverted-simpson index were relatively higher in NPKC1500 amended soils compared to other treatments and was observed to be lowest in NPKC3000 amended soils. The richness estimator showed a similar trend where NPKC3000 amended soils displayed lower species richness compared to other treatments which were nearly similar. The results are very similar with the observations by Islam MR et al. where diversity estimates
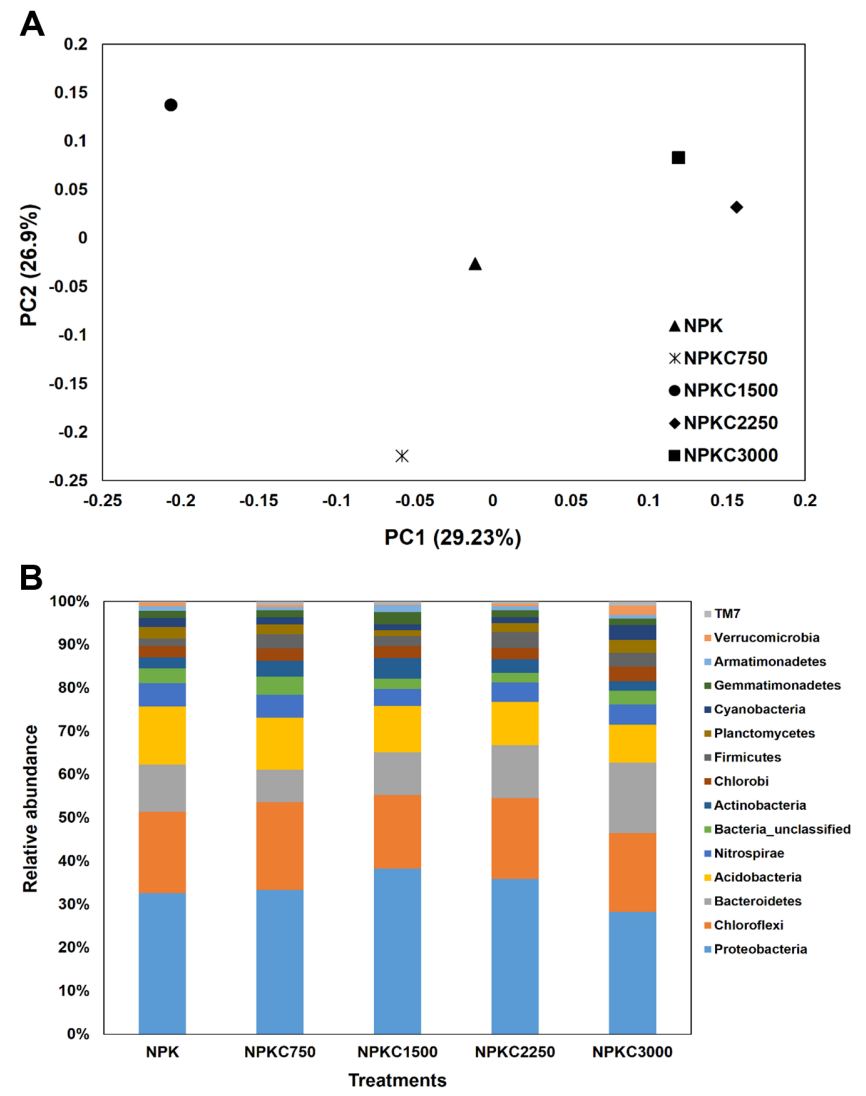

Fig. 1. (A) Principal coordinate analysis (PCoA) constructed from a Bray-Curtis dissimilarity matrix showing the bacterial community differences among the studied treatments (B). The relative abundance of bacterial community observed at phylum level. 
from community level physiological profiles (CLPP) showed lower values in higher levels of compost amended fields compared to low levels of compost application [7]. Changes in community composition among compost application levels were evident from an ordination plot (Fig. 1A) constructed from a Bray-Curtis dissimilarity matrix where higher levels of compost amended soils (NPKC2250 and NPKC3000) separated from NPK, NPKC750 and NPKC1500 along the first axis which explained 29\% of the variation in community composition. The results derive support from several studies as compost application has been reported widely to impact the size and composition of soil microbial community [8, 11, 12]. A weakness of the study was that there were no replicates of the treatments in the field and thus no replicates were added in the analyses. On that note, the results can be regarded as tentative. Nevertheless, as these fields are part of early trials established in 1954 by the Korean government to evaluate the effect of long-term fertilization, these sites are worth studying. The changes in community composition were attributed to differences in abundance profiles of certain groups of bacteria. Relative abundance of bacterial community (Fig. 1B) at phylum level showed dominant groups (> 1\%) across all the soil samples were Proteobacteria,
Chloroflexi, Bacteroidetes, Acidobacteria, Nitrospira, Actinobacteria, Chlorobi, Firmicutes, Planctomycetes, Cyanobacteria, Gemmatimonadetes, Armatimonadetes, Verrucomicrobia and TM7, which falls in line with earlier studies $[13,14]$. The most dominant phylum observed in the current study was Proteobacteria, which agrees with the earlier reports [15]. A distinct trend was observed in the relative abundance profiles of the phylum Bacteroidetes where an increase in the levels of compost induced the abundance of the particular phylum whereas abundance of Acidobacteria decreased with an increase in compost levels. At the class level (Fig. 2A), members of the bacterial phylum Bacteroidetes (Saprospirae, Bacteroidia, Sphingobacteriia, Flavobacteriia, Cytophagia) and Firmicutes (Bacilli and Clostridia) were relatively abundant in NPKC2250 and NPKC3000 when compared to other treatments. On the contrary, members of the phylum Actinobacteria, namely Thermoleophiloa, Acidimicrobiia and Actinobacteria, dominated the NPKC750 and NPKC1500 whereas their abundance decreased with an increase in levels of compost. Interestingly, members of the phylum Acidobacteria, such as iii1-8, Acidobacteria-6, BPC102, Solibacteres and Acidobacteria, were abundant in NPK amended soils and with compost application their abundance decreased
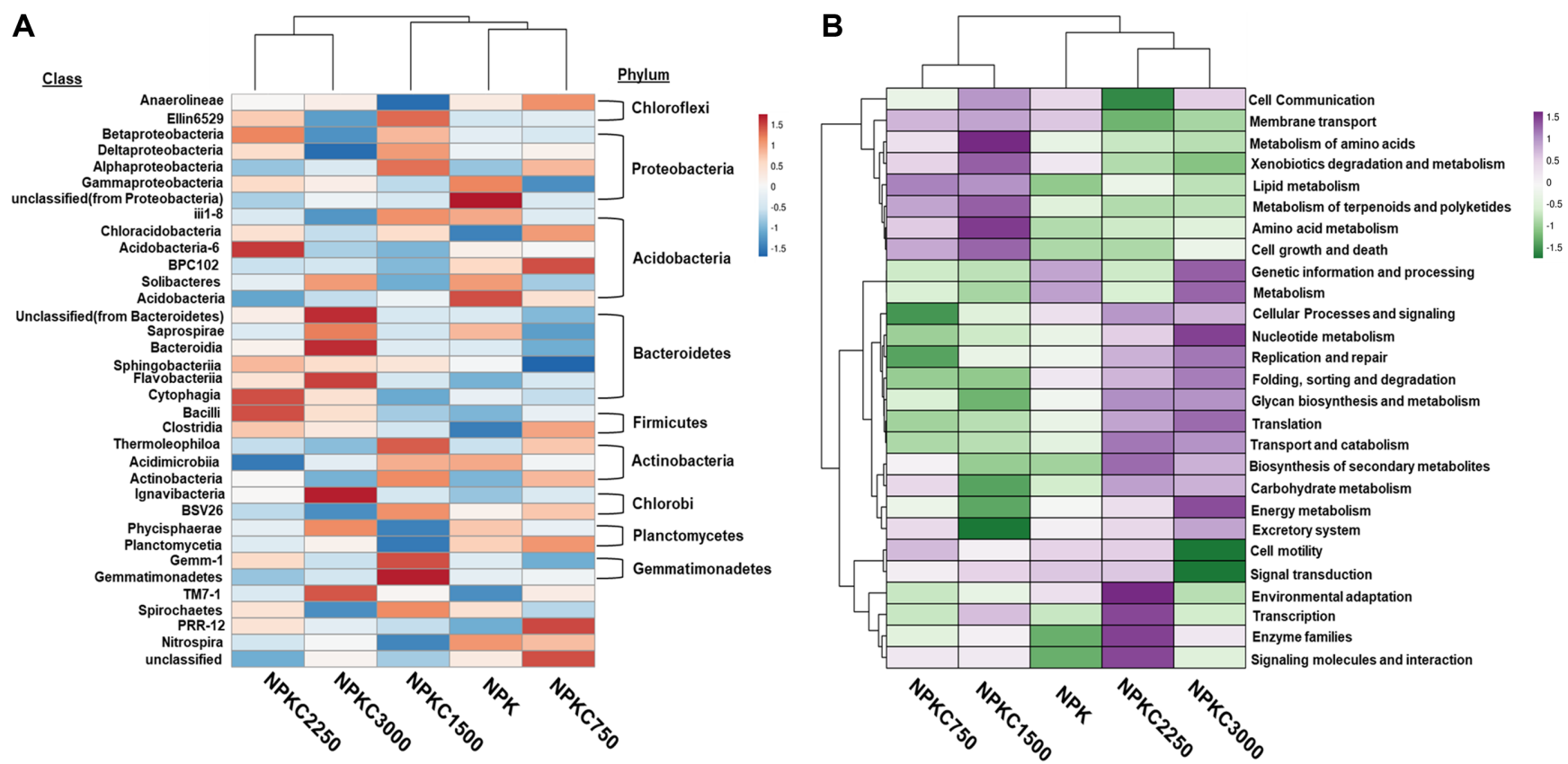

Fig. 2. (A) Heatmap illustrating relative abundance of different bacterial classes in studied treatments with the major phyla labeled. Cluster analysis indicated shifts in bacterial community in response to different levels of compost application. (B) Heatmap demonstrating clustering of studied soil samples based on relative percentage of PICRUSt-derived metabolic profiles of the bacterial community. 
considerably. Cluster analysis by Ward's method also validated the changes in bacterial community composition in response to levels of compost application. The observations are in accordance with the results from longterm fertilized paddy soil in Japan where phylogenetic analysis based on DGGE bands showed abundance of the Bacteroidetes phylum under different treatments which include rice straw and rice straw compost application in soil. From the same field, the Acidobacterial population was seen to be less abundant with no Acidobacterial members observed in soil applied with rice straw compost [16] which corroborates with our observations. Investigation of the bacterial community in cucumber plant seed and rhizosphere in response to compost application showed dominance of the Bacteroidetes population [17] which further confirms our observation. Nowadays, molecular techniques can identify microbes with key functions and this molecular information can be tuned to yield markers for evaluating soil quality [18]. Thus, identifying microbial groups and their functions has become an important task in microbial ecology [19]. On that note, PICRUSt is highly regarded because of its high quality functional predictions using marker genes, such as those of $16 \mathrm{~S}$ rRNA, and the databases KEGG (Kyoto Encyclopedia of Genes and Genomes) and COG (Clusters of Orthologous Groups of proteins) [20]. Thus, the current study employed PICRUSt to predict the functional profile of bacterial communities in the studied treatments (Fig. 2B) as compost management has been widely documented to influence the soil enzymatic activity and functionality of the microbial community [7, 21]. Clustering of the pathway abundance profiles showed bacterial community from soils with higher compost levels clustered separately from soils applied with lower levels of compost. Bacterial members originating from NPKC2250 and NPKC3000 were characterized by higher abundance of genes encoding enzymes related to nucleotide metabolism, translation machineries, replication and repair processes, carbohydrate metabolism, processes related to protein folding and general enzymatic activities compared to soils treated with low levels of compost. These results are supported by our previous observations where common soil enzymatic activities like dehydrogenase, cellulase, acid and alkaline phosphatase were significantly higher in NPKC3000 amended plots compared to other treatments [7]. On the other hand, the bacterial community inhabiting the NPK750 and NPK1500 were clustered separately and were characterized by abundance of enzymes contributing to membrane transport, amino acid metabolism and cell growth which are in line with the observations where application of farm manure is documented to increase the quantity of soil microbes [22]. The results observed imply the differential abundances of enzymes derived from differential responses of bacterial phylotypes in response to varying levels of compost. Alterations or shifts in the abundance or composition of a particular taxon as observed can explain observable conditions as well as underlying physiological processes taking place in the soil ecosystem.

\section{Acknowledgements}

The authors would like to thank the Basic Science Research Program of the National Research Foundation (NRF) under the Ministry of Education, Science and Technology (2015R1A2A1A05001885), South Korea for providing funding support towards the completion of this study.

\section{Conflict of Interest}

The authors have no financial conflicts of interest to declare.

\section{References}

1. Wu T, Chellemi DO, Graham JH, Martin KJ, Rosskopf EN. 2008. Comparison of soil bacterial communities under diverse agricultural land management and crop production practices. Microb. Ecol. 55: 293-310.

2. Lipson DA. 2007. Relationships between temperature responses and bacterial community structure along seasonal and altitudinal gradients. FEMS Microbiol. Ecol. 59: 418-427.

3. Fierer N, Jackson RB. 2006. The diversity and biogeography of soil bacterial communities. Proc. Natl. Acad. Sci. USA 103: 626-631.

4. Drenovsky RE, Steenwerth KL, Jackson LE, Scow KM. 2010. Land use and climatic factors structure regional patterns in soil microbial communities. Glob. Ecol. Biogeogr. 19: 27-39.

5. Nautiyal CS, Chauhan PS, Bhatia CR. 2010. Changes in soil physico-chemical properties and microbial functional diversity due to 14 years of conversion of grassland to organic agriculture in semi-arid agroecosystem. Soil Till. Res. 109: 55-60.

6. Islam MR, Trivedi P, Palaniappan P, Reddy MS, Sa T. 2009. Evaluating the effect of fertilizer application on soil microbial community structure in rice based cropping system using fatty acid methyl esters (FAME) analysis. World J. Microbiol. Biotechnol. 25: 1115-1117.

7. Islam MR, Chauhan PS, Kim Y, Kim M, Sa T. 2011. Community level functional diversity and enzyme activities in paddy soils under different long-term fertilizer management practices. Biol. Fertil. Soils 47: 599-604. 
8. Islam R, Trivedi P, Madhaiyan M, Seshadri S, Lee G, Yang J, et al. 2010. Isolation, enumeration, and characterization of diazotrophic bacteria from paddy soil sample under longterm fertilizer management experiment. Biol. Fertil. Soils 46: 261-269.

9. Samaddar S, Chatterjee P, Truu J, Anandham R, Kim S, Sa T. 2018. Long-term phosphorus limitation changes the bacterial community structure and functioning in paddy soils. Appl. Soil Ecol. 134: 111-115.

10. Schloss PD, Handelsman J. 2005. Introducing DOTUR, a computer program for defining operational taxonomic units and estimating species richness. Appl. Environ. Microbiol. 71: 1501-1506.

11. Pérez-Piqueres A, Edel-Hermann V, Alabouvette C, Steinberg C. 2006. Response of soil microbial communities to compost amendments. Soil Biol. Biochem. 38: 460-470.

12. Saison C, Degrange V, Oliver R, Millard P, Commeaux C, Montange $\mathrm{D}$, et al. 2006. Alteration and resilience of the soil microbial community following compost amendment: effects of compost level and compost-borne microbial community. Environ. Microbiol. 8: 247-257.

13. Ahn JH, Lee SA, Kim JM, Kim MS, Song J, Weon HY. 2016. Dynamics of bacterial communities in rice field soils as affected by different long-term fertilization practices. $J$. Microbiol. 54:724-731.

14. Feng Y, Yu Y, Tang H, Zu Q, Zhu J, Lin X. 2015. The contrasting responses of soil microorganisms in two rice cultivars to elevated ground-level ozone. Environ. Pollut. 197: 195-202.
15. Spain AM, Krumholz LR, Elshahed MS. 2009. Abundance, composition, diversity and novelty of soil Proteobacteria. ISME J. 3: 992-1000.

16. Asakawa S, Kimura M. 2008. Comparison of bacterial community structures at main habitats in paddy field ecosystem based on DGGE analysis. Soil Biol. Biochem. 40: 1322-1329.

17. Green SJ, Inbar E, Michel FC, Hadar Y, Minz D. 2006. Succession of bacterial communities during early plant development: transition from seed to root and effect of compost amendment. Appl. Environ. Microbiol. 72: 3975-3983.

18. Schloter M, Nannipieri P, Sørensen SJ, van Elsas JD. 2018. Microbial indicators for soil quality. Biol. Fertil. Soils 54: 1-10.

19. Pham VH, Kim J. 2012. Cultivation of unculturable soil bacteria. Trends Biotechnol. 30: 475-484.

20. Langille MG, Zaneveld J, Caporaso JG, McDonald D, Knights D, Reyes JA, et al. 2013. Predictive functional profiling of microbial communities using $16 \mathrm{~S}$ rRNA marker gene sequences. Nat. Biotechnol. 31: 814-821.

21. Chu H, Lin X, Fujii T, Morimoto S, Yagi K, Hu J, Zhang J. 2007. Soil microbial biomass, dehydrogenase activity, bacterial community structure in response to long-term fertilizer management. Soil Biol. Biochem. 39: 2971-2976.

22. Guanghua W, Junjie L, Xiaoning Q, Jian J, Yang W, Xiaobing L. 2008. Effects of fertilization on bacterial community structure and function in a black soil of Dehui region estimated by Biolog and PCR-DGGE methods. Acta Ecologica Sinica 28: 220-226. 